\title{
INCIDENCE AND MORTALITY OF BREAST CANCER IN WOMEN BY AGE GROUP IN BRAZIL AND LATIN AMERICA
}

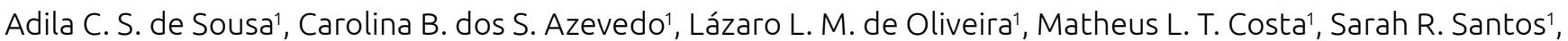
Thaynara Mercadante ${ }^{1}$

'Universidade Federal de Goiás - Goiânia (GO), Brazil.

Objective: Analyse the Latin America data on female breast cancer, comparing by age group the general rates of incidence and mortality with the Brazilian ones. Methodology: epidemiological analytic study carried out about the interpretation of data available in the GLOBOCAM stand, referring to the incidence and mortality of breast cancer in South America compared to Brazil. Results: There were 67.316 cases of breast cancer in the age group 0 to 74 years, of which 16.412 died, representing a $24 \%$ mortality rate. It was possible to identify through data analysis that the highest incidence of breast cancer is in the age group of 50-59 years, where it presented an incidence of $26 \%$, however the highest mortality rate was seen in the age group over 70 years, with a percentage of $29 \%$. In South America, excluding Brazil, it was possible to perceive that the highest incidence of breast cancer is in the age group above 70 years of age, presenting a percentage of $25 \%$ of the 48.398 cases. Conclusion: From these data it is possible to conclude that the mortality rate does not follow the same age pattern of the incidence rate, that is, there are variations according to age. Such variations reveal that the higher the age group, the higher the mortality rate. This can be caused by the fact that cancer, in this age group, presents a histological grade of higher malignancy, either by a late detection or by the patient's advanced age. Thus, early diagnosis is of great importance for reducing mortality rates and intervening in factors that influence detection is fundamental, such as: accessibility to the health service, coverage of mammography and quality of treatment. 